\title{
An ensemble based approach using a combination of clustering and classification algorithms to enhance customer churn prediction in telecom industry
}

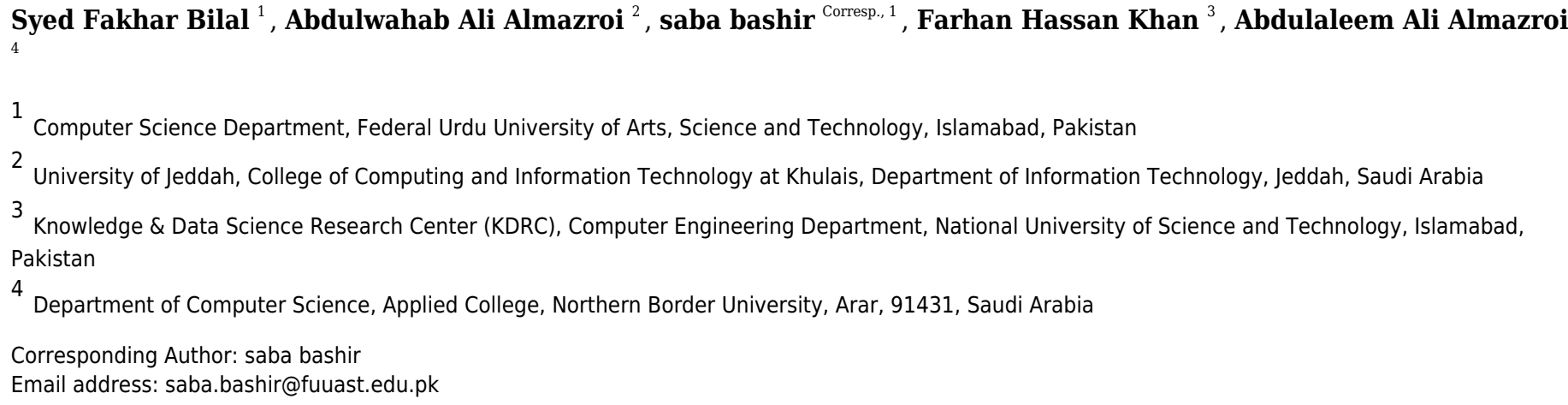

Mobile communication has become a dominant medium of communication since the past two decades. New technologies and competitors are emerging rapidly and churn prediction has become a great concern for telecom companies. A customer churn prediction model can provide an accurate identification of potential churners so that a retention solution may be provided to them. The proposed churn prediction model is a hybrid model that is based on combination of clustering and classification algorithms using ensemble. First, different clustering algorithms i.e K-means, K-medoids, X-means and Random clustering are evaluated individually on two churn prediction datasets. Then hybrid models are introduced by combining clustering with seven different classification algorithms individually and then evaluation is performed using ensembles. The proposed research is evaluated on two different benchmark telecom data sets obtained from GitHub and Bigml platforms. The analysis of results indicates that the proposed model has attained highest prediction accuracy of $94.7 \%$ on GitHub dataset and $92.43 \%$ on Bigml dataset. State of the art comparison is also performed with proposed model. The proposed model significantly performs better than state of the art churn prediction models. 
2 An ensemble based approach using a combination of 3 Clustering and Classification Algorithms to Enhance 4 Customer Churn Prediction in Telecom Industry

5

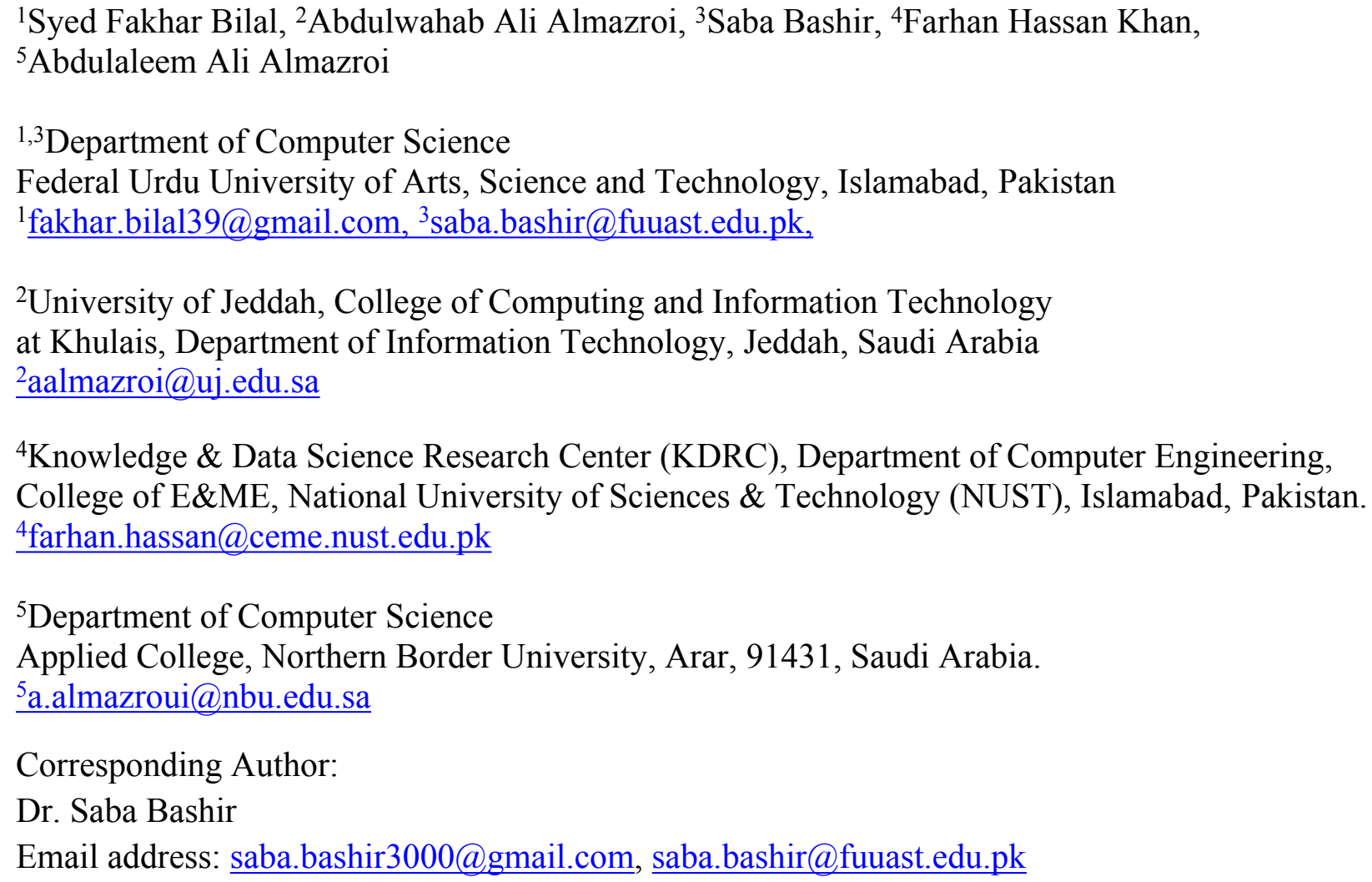

${ }^{4}$ Knowledge \& Data Science Research Center (KDRC), Department of Computer Engineering, College of E\&ME, National University of Sciences \& Technology (NUST), Islamabad, Pakistan. 4farhan.hassan@ceme.nust.edu.pk

${ }^{5}$ Department of Computer Science Applied College, Northern Border University, Arar, 91431, Saudi Arabia. 5a.almazroui@nbu.edu.sa

Corresponding Author:

Dr. Saba Bashir

Email address: saba.bashir3000@gmail.com, saba.bashir@,fuuast.edu.pk

\begin{abstract}
Mobile communication has become a dominant medium of communication since the past two decades. New technologies and competitors are emerging rapidly and churn prediction has become a great concern for telecom companies. The proposed churn prediction model is a hybrid model that is based on combination of clustering and classification algorithms using ensemble. First, different clustering algorithms i.e K-means, K-medoids, X-means and Random clustering are evaluated individually on two churn prediction datasets. Then best clustering method is selected among them to combine with classification algorithms. Next, hybrid models are introduced by combining selected clustering method with seven different classification algorithms individually and then evaluation is performed using ensembles. Finally, proposed model is selected based on highest performance on all datasets which is K-medoid+Gradient Boosting Tree+Decision Tree+Deep Learning + AdaBoost. The proposed research is evaluated on two different benchmark telecom data sets obtained from GitHub and Bigml platforms. The analysis of results indicates that the proposed model has attained highest prediction accuracy of $94.7 \%$ on GitHub dataset and $92.43 \%$ on Bigml dataset. State of the art comparison is also
\end{abstract}


performed with the proposed model. The proposed model significantly performs better than state of the art churn prediction models.

Keywords- Churn Prediction, hybrid models, telecommunication data mining, classification, clustering, prediction model

\section{Introduction}

Data mining is the way of identifying patterns and extracting knowledge from large amount of data [1]. It allows us to identify the future flow employing different prediction models. Enterprises can predict future patterns and trends using different data mining tools and techniques. The purpose of data mining is to analyse meaningful information that is hidden in huge amounts of datasets and incorporate such information for useful tasks [63].

Telecom sector is becoming one of the most important industries in developed countries since the past two decades [2]. Data mining plays a vital role for prediction and analysis in the telecom industry due to availability of huge data. The basic application area is to perform prediction of churner in order to save customer retention and to make a high-profit rate. Data mining techniques are used in the telecom sector to observe the churn behaviour of the customers. With the increasing rate in users of telecom companies, they now offer variety of services for the retention of customers. In order to obtain better services and benefits, the customer switches its service provider and the phenomenon is known as churn. If a customer switches a service provider's company then face loss occurs in the company's revenue. Prediction can be performed to identify the potential churners and retention solutions may be provided to them. A large number of mining algorithms are available which classify the behavior of customers into churner and non-churners.

Telecom sector is growing rapidly due to different technologies. Different companies provide quality of data for communication; some gives better services as compared to others. In order to stop churn, companies offer different services which are attractive for their customers. Data mining technologies are used to perform churn prediction using different algorithms like Naïve Bayes, Decision tree, Neural network and Logistics regression etc. An accurate prediction model is very helpful for correct identification of customer's churn and plays a vital role in making decisions about their retention [3].The best customer churn prediction model can identify churner and gives directions to decision-makers for generating maximum profit [4-6]. There are number of reasons for which churning of customers. Most important of them are calls or packages rate which do not suit the customer $[9,10]$. We can identify weather a customer wants to leave or not on the basis of his historical data and behavior.

Existing studies show that an efficient churn prediction model should efficiently use large volume of historical data in order to perform churner's identification. However, there are number of limitations in existing models due to which it is not possible to perform churn prediction efficiently and with high accuracy. A large volume of data is generated in telecom sector which contains missing values. Prediction on such type of data results in poor/inaccurate outputs for prediction models in literature. Data preprocessing is now performed to resolve this issue and missing values imputation is performed using machine learning methods which results in high performance and classification/prediction accuracy. Feature selection is also performed in literature however some important and information rich features are neglected during model development. Moreover, statistical methods are used for model generation which results in poor prediction performance. Furthermore, benchmark datasets are not used for model evaluation in literature resulting in poor representation of true picture of data. Fair comparison between 
87

different models cannot also be performed without benchmark datasets. An intelligent model can be used to resolve the existing issues and to provide churn prediction more accurately. The proposed churn prediction model is based on combination of clustering and classification algorithms. The performance of proposed model is evaluated on different churn prediction datasets. The evaluation of proposed churn prediction model is evaluated using different metrics such as accuracy, precision, recall and f-measure. The objectives of proposed research are; to identify the issues in literature and provide an efficient model for customer churn prediction, to identify the churners with high accuracy. The retention strategies may then be provided to the potential churners. It is also observed from the experiments that proposed churn prediction model performed better in terms of churn prediction by achieving high accuracy.

The main contributions of this research are as follows:

- Proposed a churn prediction model with high accuracy

- Data preprocessing is performed for missing values imputation, noise removal and duplicates removal

- Selection of important features using feature selection technique

- Combination of clustering and classification techniques to perform customer churn prediction on two large datasets of telecom sector

- Customer profiling is performed using clustering technique to divide the behavior of customer into different groups like low, medium and risky

The remaining organization of paper is as follows: section II provides literature review. Section III presents the proposed churn prediction model. Experimental evaluation and results are presented in section IV. Finally, section V presents the conclusion and future work.

\section{Literature Review}

Irfan Ullah et al. [2] proposed a churn prediction model named JIT-CCP model. In this model first step is data pre-processing, second step is binary classification and evaluation of performance by using the values of confusion matrix corresponding to true positive, true negative, false positive and false negatives. Based on these terms, probability of detection is calculated. Probability of detection (PD) is used to calculate the accuracy of multiple classifiers. If PD value is near to 1 then the classifier's results are much better and vice versa. However, the proposed model is not suitable for a large amount of data.

J. Vijaya et al. [3] presented that customer retention plays a valuable role in the success of a firm. It not only increases company's profit but also maintains company's ranking among telecom industry. Customer retention is less costly rather than making new customers. So maintenance of the customers and customer association management (CAM) are the two parameters for the success of every company. In this research hybrid model of supervised and unsupervised techniques are used for churn prediction. There are different stages of this modal. In the first stage, data is cleaned and removed different deviations from data. In next stage, testing and training sets of data are obtained from different clusters. After this, prediction algorithms are applied. In the final stage accuracy, specificity and sensitivity are measured for evaluating the efficiency of proposed model.

Höppner et al. [4] stated that customer retention policies rely on different predictive models. The most recent development is expected to maximize the profit (EMPC) which selects the most valuable churn model. In this research a new classification method has been introduced, which integrates the (EMPC) matrix directly to churn model. This technique is known as ProfTree. The main advantage of this model is that a telecom company can gain maximum profit. The proposed 
133

134

135

136

137

138

139

140

141

142

143

144

145

146

147

148

149

150

151

152

153

154

155

156

157

158

159

160

161

162

163

164

165

166

167

168

169

170

171

172

173

174

175

176

177

model has increased performance and accuracy as compared to other models. In future this model may be combined into different algorithms to further increase the prediction performance. Ali et al. [5] used different mining algorithms and techniques for prediction of churners. WEKA software is used for applying different classifiers. The first step of this model is data preprocessing where missing values are removed. After preprocessing, FSS (Feature Subset Selection) steps are performed where feature reduction is performed. It also reduced cost of securing the data. After that, information Gain Ratio is performed to rank the target dataset. The advantage of this research is to identify interesting patterns for prediction of churner's behaviour. The disadvantage of this research is that if dataset will be increased then the process will become slow and requires more time for prediction.

Bharat et al. [26] proposed a model based on the activity pattern of customers. Specifically, its measurement is based on the customer's activity by finding the average length of inactive time and frequencies of inactivity. The proposed method can be used in other domains for churn prediction.

Gajowniczek et al. [27] used Artificial Neural Network with entropy cost functions for prediction model of customers. Numerical method like classifications tree or SVM provides higher accuracy in classification, which shows the simplest way to apply the new q-error functions to conclude the issue. Runsha Dong et al. [41] stated that customer churn is valuable for telecom companies to retain weighty users. A customer ccp model having more accuracy is very weighty for decision of customer retention. In this paper SVM technique is also used because it is much better for precision. It solves samples under low dimensional space which is linear inspirable in two dimensional space. There is a limitation of proposed model like it is very difficult to quantify churned customers. Therefor there should more complex investigation.

Ahmed et al. [28] proposed a model based on combination of different classifiers in order to create hybrid ensembles model for prediction. In this paper bagged stack learners are proposed. Experimentation is performed on two datasets related to telecom companies. High accuracy is obtained. The benefit of this model is that it does not work on generalized data sets. Brownlow et al. [29] introduced a new methodology for churn prediction in fund management services and implementation. This framework is based on ensembles learning and a new weighting mechanism is proposed to deal with imbalanced cost sensitivity problem with financial data. In this model heterogeneous type of data are used collected from different companies. The performance of this model may be increased with extraction and enhancement of learning methods.

$\mathrm{VO}, \mathrm{N}$ et al. [30] used text mining and data mining methods for the prediction of churn. Multiple methods are used for the prediction of churns like semantic information and word importance. This model uses only unstructured data for prediction. In future this research can be extended into segmentation and building personalized recommendation system for different financial services and products.

Calzada-Infante et al. [31] performed comparison of two techniques Time-Order-Graph and Aggregated-Static-Graph with forest classifier using three threshold measures to evaluate the predictive performance of the similarity forest classifier with each centrality metric.

Nguyen, N. et al. [32] discussed the comparison of two prediction techniques which are SMOTE and Deep Belief Network (DBN) against two cost sensitive learning approaches e.g Focal Loss and Weighted Loss. The results show better performance of Focal Loss and Weighted Loss than SMOTE and DBN. 
Vural, $\mathrm{U}$ et al. [33] proposed a new method based on ANN for churn prediction. In this method two layers of ANN are used to predict churn.

Jain, $\mathrm{H}$ et al. [34] discussed overview of different classification algorithms. These algorithms are Multi-Layer-Perception, KNN measure, Fuzzy Cluster and Deep Learning CNN. After comparison of results Deep Learning CNN shows better results as compared to other classification algorithms.

Although a lot of work has been done on churn prediction but still there is room for improvement. There is a need of churn prediction model which has high prediction accuracy. The proposed modal is based on combination of clustering and classification techniques and attained high prediction accuracy. The summary of literature is shown in Table 1.

\section{Proposed Methodology}

The proposed model has increased the churn prediction performance by using a hybrid model where combinations of different clustering and classification ensembles are introduced. Figure 1 shows different modules of proposed model.

\subsection{Data Acquisition}

The first task of proposed model is data acquisition. Two benchmark churn prediction datasets have been used for model evaluation. These datasets are acquired from online data repositories. First churn prediction dataset is collected from GITHUB which is freely available online data repository for research. This dataset contains 5000 instances with 707 churn customers and 4293 non-churn customers. The second dataset is collected from Bigml platform which is also freely available online repository containing 3333 instances with 21 attributes having 483 churns and 2850 non-churn values. This dataset contains the information about customer's concerns behavioural, demographics and revenue information.

\subsection{Data Preprocessing}

The main purpose of data pre-processing is to remove noise, anomalies, missing values and duplication from data [36]. In pre-processing, a model needs to remove missing values, noisy data, duplication and only needs to use important features from data [66]. Data preprocessing is the first step which is applied on churn prediction data. The proposed churn prediction model has incorporated following tasks during data pre-processing.

- Data Cleaning: Prediction is very difficult when there are missing values, duplication and noise in the data. So data cleaning is performed to replace missing values with actual values which are calculated by each attribute mean, remove duplicated data and noise/error values are identified and removed.

- Feature Selection: Feature selection is the most important step of data pre-processing. Feature selection is performed using forward selection and most important features are chosen for prediction model.

- Data Reduction: In this step data is reduced in smaller volume for producing compact and understandable results. 
216

217

218

219

220

221

222

223

224

225

226

227

228

229

230

231

232

233

234

235

236

237

238

239

240

241

242

243

244

245

246

247

248

249

250

251

\subsection{Clustering Algorithms}

After data preprocessing, clustering is applied on cleaned and refined data. The proposed model has employed clustering in order to improve the prediction performance. Following clustering methods have been used by the proposed model.

\subsubsection{K-Means Clustering}

$\mathrm{K}$ Means clustering algorithm divides $\mathrm{N}$ rows into $\mathrm{K}$ segments, and $\mathrm{K}$ is always less than $\mathrm{N}$. It randomly selects the value of $\mathrm{k}$ which represents centre of cluster mean. It measures the distance between the clusters and compute mean for every cluster. The process continuous iteratively until desired clusters is refined. Following formula is used to measure the distance [27].

$J(V)=\sum_{i=1}^{c} \sum_{j=1}^{c_{i}}\left(\left\|x_{i}-v_{j}\right\|\right)^{2}$

Where $\left\|\mathrm{x}_{\mathrm{i}}-\mathrm{v}_{\mathrm{j}}\right\|$ is the Euclidean distance between two clusters.

\subsubsection{K-Medoids Clustering}

In 1987, Rousseeuw Lloyd and Kaufman introduced a clustering technique which is also partitioned based and is termed as K-Medoids algorithm. K-Medoids is more robust to noise and outliers as compared to k-means [27]. Following formula is used to calculate the cost of each cluster.

$$
c=\sum_{c_{i}} \sum_{p_{i} \in c_{i}}\left|P_{i}-C_{i}\right|
$$

Where $\mathrm{P}_{\mathrm{i}}$ and $\mathrm{C}_{\mathrm{i}}$ are objects for which dissimilarity is calculated.

\subsubsection{X-Means Clustering}

$\mathrm{X}$ means clustering is a variation of $\mathrm{k}$ means clustering where clusters are refined and subdivided repeatedly until Bayesian Information Criteria (BIC) is reached. The efficient estimation of number of clusters is obtained automatically instead of take input from user in the form of K. Covariance of each cluster is measured and following formula is used to calculate the variance [27].

$\sigma^{2}=\frac{1}{R-K} \sum_{i}\left(x_{i}-\mu_{(i)}\right)^{2}$

Where $\mathrm{R}$ and $\mathrm{K}$ are number of points and number of clusters respectively and $\mu$ is the centroid of i cluster.

\subsubsection{Random Clustering}

Random clustering is often used in Rapidminer to perform random flat clustering of given dataset. Moreover, some of the clusters can be empty and the samples are assigned to clusters randomly.

\subsection{Classification and Prediction Algorithms}

After performing clustering, Classification is performed by the proposed model. Each clustering method is evaluated and best clustering method is combined with classification algorithms. The proposed model first used single classifiers and their performance is measures. Then, ensemble 
252 classifiers are used along with clustering to attain the highest prediction accuracy. The 253 combination of clustering and ensemble classifier which has attained highest churn prediction 254 accuracy will be considered as proposed model. Following classifiers are used by the proposed 255 churn prediction model.

$256 \quad 3.4 .1$ K-Nearest Neighbor $(\mathrm{kNN})$

257 The k-nearest neighbor is one of the simplest classification methods in data mining. Following 258 distance formula is used to measure the distance [27]:

$259 d(x, y)=\sqrt{\sum_{i=1}^{k}\left(x_{i}-y_{i}\right)^{2}}$

260 Where $\mathrm{k}$ is number of samples in training data $\mathrm{x}$ and $\mathrm{y}$ are instances for which distance is

261 calculated.

262 3.4.2 Decision Tree

263 Quinlan introduced in 1993 a divide and conquer method and termed as decision tree. Entropy

264 and then information gain is calculated for each attribute using the following formulas.

265

$H(S)=\sum_{x \in X}-p(x) \log _{2} p(x)$

266 Where $\mathrm{S}$ is the dataset, $\mathrm{X}$ is set of classes in $\mathrm{S}$ and $\mathrm{p}(\mathrm{x})$ is probability of each class.

267

$I G(S, A)=H(S)-\sum_{t \in T} p(t) H(t)=H(S)-H(S \mid A)$

268

269

270

271

272

273

274

275

276

277

278

279

280

281

282

Where $\mathrm{H}(\mathrm{S})$ is entropy of $\mathrm{S}, \mathrm{T}$ is the subset of $\mathrm{s}, \mathrm{p}(\mathrm{t})$ is probability of subset $\mathrm{t}$ and $\mathrm{H}(\mathrm{t})$ is entropy of subset $t$.

\subsubsection{Gradient Boosted Tree (GBT)}

The idea behind GBT is to improve the prediction accuracy by producing ensemble of decision trees. GBT outperforms random forest as it produces the ensemble of weak prediction models.

The prediction is given as follows:

$\hat{y_{i}}=\sum_{j} \theta_{j} x_{i j}$

Where $\mathrm{y}$ is the prediction made by input $\mathrm{x} . \Theta$ is the best parameter that best fits the model.

\subsubsection{Random Forest (RF)}

Random forest or random decision forest is an ensemble learning method used for classification, regression and other tasks that operate by constructing a multitude of decision tree at training time and outputting the class that is the mode of the classes or average prediction (regression) of the individual trees. Entropy or Gini index are used for tree construction using following formulas.

Gini $=1-\sum_{i=1}^{c}\left(p_{i}\right)^{2}$ 
283 Entropy $=\sum_{i=1}^{c}-p_{i} * \log _{2} p_{i}$

284

285

286

287

288

289

290

291

292

293

294

295

296

297

298

299

300

301

302

303

304

305

306

307

308

309

310

311

312

313

314

315

\subsubsection{Deep Learning (DL)}

It is a multi-layer technique which compares large number of layers of neurons. It is an artificial network which is used to solve more complex and difficult problems of data mining.

\subsubsection{Naïve Bayes}

Naive Bayes method is a supervised learning algorithm based on applying Bayes' theorem. Following formula is used by the Naïve Bayes classifier.

$P(c \mid x)=\frac{P(x \mid c) P(c)}{P(x)}$

Where $\mathrm{P}(\mathrm{c})$ is the prior probability of class, $\mathrm{P}(\mathrm{x})$ is prior probability of predictor, $\mathrm{p}(\mathrm{x} \mid \mathrm{c})$ is probability of predictor given class and $\mathrm{p}(\mathrm{x} \mid \mathrm{c})$ is posterior probability of class.

\subsubsection{NB (K) (Naïve Bayes Kernel)}

The Naive Bayes (Kernel) operator can be applied on numerical attributes. A kernel is a weighting function used in non-parametric estimation techniques. Kernels are used in kernel density estimation to estimate random variables' density functions, or in kernel regression to estimate the conditional expectation of a random variable.

\subsubsection{Ensemble classifiers}

Krawczyk, B. et al. [37] used ensemble methods to apply multiple learning algorithms for prediction. Ensembles increase the performance of the system or model [64].

Following ensemble models are used by the proposed model.

\subsubsection{Voting}

Voting method is used to combine the results of individual classification algorithms using majority voting. Each individual classifier assigns a class label to test data, then their results are combined using voting and final class prediction is generated using maximum number of votes for a particular class [55]. Following formula is used to apply majority voting on dataset:

$\sum_{t=1}^{T} d_{t, J}(x)=\max _{j=1,2,3 \ldots \ldots . . C} \sum_{t=1}^{T} d_{t, J}$

Where T represents the number of classifiers, and $d(t, J)$ is the decision of classifier and $\mathrm{J}$ represents the classes.

\subsubsection{Bagging}

Bagging stands for Bootstrap Aggregation. It is an ensemble classifier which has bag of similar and dissimilar objects. It helps to decrease the variance of the classifiers which are used in prediction model to make better performance [56]. Then evaluation of Bagging is given as follows: 
$316 V_{t, j}=\left\{\begin{array}{rr}1 & \text { if } h_{t} \text { picks class } w_{j} \\ 0 & \text { otherwise }\end{array}\right.$

317 Where $t$ represents training samples, $h_{t}$ represents trained classifiers and $\mathrm{w}_{\mathrm{i}}$ represents class

318 labels. Each class will have total votes represented by:

319

$V_{j}=\sum_{t=1}^{T} v_{t, j} j=1,2,3 \ldots \ldots . . c$

320

321

322

323

324

325

326

327

328

329

330

331

332

333

334

335

336

337

338

339

340

341

342

343

344

345

346

347

348

349

350

351

352

353

354

\subsubsection{AdaBoost}

AdaBoost is the short form of Adaptive Boosting, is a Meta algorithm, which can be used in conjunction with different other learning algorithms to improve their performance [56].

Weighted majority voting is applied on the classifiers. Every classifier gets equal opportunity to draw samples in each iteration. Following formula is used to apply weighted majority voting:

$V_{j}=\sum_{t: h_{t}(x)=w_{j}} \log \left(\frac{1}{\beta_{t}}\right) \quad j=1,2,3 \ldots \ldots \ldots \ldots$

Where $\beta_{t}$ is normalized error, $\mathrm{h}_{\mathrm{t}}$ represents trained classifiers and $\mathrm{w}_{\mathrm{j}}$ represents class labels of training data.

\subsubsection{Stacking}

Stacking is used for combining different leaners rather than selecting among them. It can be used for getting a performance better than any single one of the trained models. Bootstrapped samples of training data are used to train the classifiers. There are two types of classifiers used in stacking, Tier-1 classifiers and Tier-2 classifiers. Tier-1 classifiers are trained on bootstrapped samples and generate prediction, their result are then used to train Tier-2 classifiers. This way training data is properly used to perform learning [56].

\subsection{Working of Proposed Model}

The proposed model has increased the churn prediction performance by using a hybrid model where clustering methods and classification methods are combined. Combinations of different clustering and classification ensembles are introduced and best combination models are selected for final prediction. First clustering is used to generate the clusters of given dataset. "Map clustering on Label" operator is used to assign labels to data. Then classification is performed for labelled data to generate the results.

It is also proved that performance; accuracy and efficiency of churn prediction model can be increased by using the proposed novel hybrid models. As single classifier based model cannot provide high accuracy, therefore the proposed models used the hybrid model for prediction of churn.

1. First of all clustering evaluation is carried out and results are obtained and select best clustering technique on the behalf of accuracy.

2. After clustering, single classifier based classification is performed and then accuracy, precision, recall and f-measure results are obtained.

3. After that hybrid model of best clustering method and each single classifier is developed and performance results are obtained for each dataset.

4. Next, only ensemble classifiers based models are developed and evaluated on both datasets. 
5. Then, these ensemble models are combined with best clustering technique in order to make hybrid models and performance is evaluated. It is clear from the evaluation that proposed combination of clustering and ensemble models has achieved highest prediction accuracy as compared to state of the art models for both churn prediction datasets.

\section{Experimental Results, Evaluation and Discussion}

The experimental of proposed model is performed on two benchmark churn prediction datasets. First, clustering techniques are performed on each dataset and best clustering method is selected. Next, single classifiers are performed on each dataset and their performance is evaluated, shown in section 4.5. Then, single classifiers are evaluated along with K-Medoid clustering and again their performance is evaluated for each dataset as shown in sections 4.6. It is analyzed that performance of single classifiers is improved. Afterthat, ensembles (Voting, Bagging, Stacking, AdaBoost) are evaluated on each dataset along with KMedoid clustering as shown in sections 4.7. The analysis indicates that AdaBoost ensemble along with clustering performed better as compared to other ensembles for both churn prediction datasets.

Following datasets are used for the experiments and evaluation which are freely available at online data repositories.

\subsection{GITHUB Dataset}

First churn prediction dataset is collected from GITHUB which is an online data repository. The datasets are freely available over here for research. The dataset name is "Kaggle-telecomcustomer-churn-prediction" obtained from the data source https://www.kaggle.com/blastchar/telco-customer-churn. It is used to predict customer's behaviour to retain them. It contains 5000 instances data where each row represents a customer and columns represents customer's attributes. The dataset contains 707 churn customers and 4293 non-churn customers.

\subsection{BigML Dataset}

The second dataset is collected from Bigml platform which is also freely available online data repository. The dataset is obtained from data source https://cleverdata.io/en/bigdata-predictionsbigml/. The name of dataset is "Churn in Telecom's dataset". It contains 3333 instances having 21 attributes. There are 483 churns and 2850 non churn customers in the dataset. This dataset is also used to predict the customer's behaviour.

\subsection{Rapid Miner}

Rapid Miner is a data science software platform that provides an integrated environment for data preparation, machine learning, deep learning, text mining, and predictive analytics. The proposed research is implemented using Rapid Miner. It is also freely available on the web.

\subsection{Model Evaluation}

Confusion matric is used for model evaluation. With the help of confusion matrix performance of proposed model is analysed. The performance of proposed model is analysed using accuracy, precision, recall and f-measure $[61,62,65]$. These parameters can be measured with the help of following formulae where TP represents true positives, TN is true negatives, FP shows false positives and FN shows false negatives. 
395

396

397

398

399

400

401

402

403

404

405

406

407

408

409

410

411

412

413

414

415

416

417

418

419

420

421

422

423

424

425

426

427

428

429

430

431

432

433

$$
\text { Accuracy }=\frac{T P+T N}{T P+T N+F P+F N}
$$

$$
\text { Precision }=\frac{T P}{T P+F P}
$$

$$
\operatorname{Re} \text { call }=\frac{T P}{T P+F N}
$$

$$
F-\text { Measure }=\frac{2 * \operatorname{Pr} \text { ecision } * \operatorname{Re} \text { call }}{\operatorname{Pr} \text { ecision }+\operatorname{Re} \text { call }}
$$

\subsection{Map Clustering on Label}

After clustering, mapping is used to generate TP, TN, FN, and FP from datasets. Mapping maps the cluster 0 and cluster 1 with churner and non-churner i.e 0 and 1. For example we have a table containing three columns cluster0, cluster 1 and churn; mapping generates another column prediction (churn) which contains class 0 and class 1 . It maps the values of cluster 0 and clusters 1 with class 0 and class 1 and then TP, TN, FN, and FP can be generated.

After applying clustering technique on dataset, there are two clusters which are cluster_0 and cluster_1. These clusters are mapped with prediction class and check whether the values of class lie in clusters or not. So for this purpose clusters are mapped with Prediction (Class) and analysed. If values of cluster_0 lie in prediction class 0 , it generates TN, and if values of cluster 1 lie in Prediction (Class) 1 it generates TP. Similarly, if values of cluster_0 lies in Prediction (class) 1 it generates FP, and if values of cluster_1 lies in Prediction (Class) 0 it generates FN. Therefore, cluster evaluation is performed with the help of mapping. Table 2 shows clustering evaluation results on GITHUB and Bigml datasets.

\subsection{Clustering with Single Classifier on Churn Prediction Datasets}

As it is clear from the literature that single classification techniques show low classification accuracy as compared to hybrid model, therefore now supervised and unsupervised techniques are combined to generate hybrid model and then this hybrid model will be used for classification in order to increase the accuracy level. It is analysed from table 2 that k-med shows higher accuracy as compared to other clustering techniques therefore now the combination of $\mathrm{k}$-med with seven different classification algorithms (GBT, DT, RF, kNN, DL, NB, NB(K)) is applied one by one on each dataset as shown in table 3 .

\subsection{Clustering with Ensembles on Churn Prediction Datasets}

Tables 4-7 show that k-med clustering is combined with different combination of classifiers. Voting, Bagging, Stacking and AdaBoost ensembles are used. The combination of GBT, DT and DL shows highest accuracy when it is combined with k-med clustering.

\subsection{Comparison of Different Techniques}

Now the comparison of all techniques has been carried out. The comparison shows different levels of accuracy for different hybrid models. The average accuracy of different techniques has 
434

435

436

437

438

439

440

441

442

443

444

445

446

447

448

449

450

451

452

453

454

455

456

457

458

459

460

461

462

463

464

465

466

467

468

469

470

471

472

473

474

475

476

477

been compared. Table 8 shows the comparison results. As it is clear from experiments that results are improved on each step because a hybrid approach is used to improve the results.

\subsection{Comparison with State of the Art Techniques}

Table 9and table 10 show the comparison of proposed model with different state of the art techniques. Proposed model shows higher accuracy as compared to existing techniques. In this research hybrid models of supervised and unsupervised learning techniques is proposed and implemented with rapid miner. These models are applied on two datasets which are freely available on online data repositories. In first step clustering algorithms are selected i.e k-means, $\mathrm{K}$-medoid, X-means and Random Clustering are selected for experimentation. After Evaluation of these clustering algorithms it is noticed that the k-medoid showed high accuracy as compered other three clustering algorithms, so K-medoid is selected for hybrid model generation. After selection of clustering algorithm next step is selection of classification algorithm. Seven different classification algorithms are selected based on literature which is GBT, DT, RF, KNN, DL, NB, NB (K) for classification. These algorithms have high performance for churn prediction datasets. After evaluation it is noticed that GBT shows high accuracy level. After separate single experimentation a hybrid model of clustering and single classification algorithm is developed to perform the results. In this experimentation K-med and GBT shows better accuracy as compared to other combinations. After combination of single classification algorithm and clustering a hybrid model of classification algorithms is implemented and experiments are performed. The main reason of implementation of hybrid model is that it shows better accuracy as compared to single classifiers [53,54], so different combination of above mentioned classifiers are used for experimentation. These hybrid classifiers are used with k-med clustering for better results. After this step Ensemble classifiers voting, bagging, adaBoost and stacking are used with hybrid model of clustering and classification. These ensemble classifiers are used to increase the accuracy level. With the combination of ensemble classifiers and clustering algorithm, models show better accuracy for churn prediction.

\section{Conclusion and Future Work}

Customer churn prediction is a critical problem for the telecom companies. The identifying of customers that are not happy with the services allow the companies to work on their weak points, pricing plans, promotions and customer preferences to reduce the reasons for churn. Many techniques are used in literature to predict customer's churn. The proposed research focused on introducing different models for customer churn prediction with high accuracy. Novel hybrid models are introduced by combining clustering and classification approaches. Then the proposed models are evaluated on two churn prediction datasets obtained from online data repositories. The analysis of results show that proposed models have achieved higher classification and prediction accuracy as compared to existing state of the art models. In this work the combination of k-med clustering and GBT, DT and DL classifier ensemble shows higher accuracy as compared to other methods.

This research can be extended in future by using big data analytics. Social network analysis can be used to identify the customer's satisfaction level towards telecom services and then these services can be offered to reduce the churn rate. Further datasets can also be used to increase the confidence level on results. Finally, the models can be applied on different sectors like banking, insurance or airline and prediction accuracy can be compared.

Peer] Comput. Sci. reviewing PDF | (CS-2021:07:64230:1:1:NEW 1 Dec 2021) 
478

479

480

481

482

483

484

485

486

487

488

489

490

491

492

493

494

495

496

497

498

499

500

501

502

503

504

505

506

507

508

509

510

511

512

513

514

515

516

517

518

519

520

521

522

\section{References}

[1] Han, J., Pei, J., \& Kamber, M. (2011). Data mining: concepts and techniques. Elsevier. [2] Ullah, I., Raza, B., Malik, A. K., Imran, M., Islam, S. U., \& Kim, S. W. (2019). A churn prediction model using random forest: analysis of machine learning techniques for churn prediction and factor identification in telecom sector. IEEE Access, 7, 60134-60149.

[3] Vijaya, J., \& Sivasankar, E. (2018). Improved Churn Prediction Based on Supervised and Unsupervised Hybrid Data Mining System. In Information and Communication Technology for Sustainable Development (pp. 485-499). Springer, Singapore., [4] Höppner, S., Stripling, E., Baesens, B., \& Verdonck, T. (2017). Profit Driven Decision Trees for Churn Prediction. arXiv preprint arXiv:1712.08101.

[5] Ali, M., Rehman, A. U., Hafeez, S., \& Ashraf, M. U. (2018, August). Prediction of Churning Behavior of Customers in Telecom Sector Using Supervised Learning Techniques. In 2018 International Conference on Computer, Control, Electrical, and Electronics Engineering (ICCCEEE) (pp. 1-6). IEEE.

[6] Amin, A., Shah, B., Khattak, A. M., Moreira, F. J. L., Ali, G., Rocha, Á., \& Anwar, S. (2019). Cross-company customer churn prediction in telecommunication: A comparison of data transformation methods. International Journal of Information Management, 46, 304-319. [7] Ahmed, A., \& Linen, D. M. (2017, January). A review and analysis of churn prediction methods for customer retention in telecom industries. In 2017 4th International Conference on Advanced Computing and Communication Systems (ICACCS) (pp. 1-7). IEEE.

[8] Franciska, I., \& Swaminathan, B. (2017, May). Churn prediction analysis using various clustering algorithms in KNIME analytics platform. In 2017 Third International Conference on Sensing, Signal Processing and Security (ICSSS) (pp. 166-170). IEEE.

[9] Tiwari, A., Sam, R., \& Shaikh, S. (2017, February). Analysis and prediction of churn customers for telecommunication industry. In 2017 International Conference on I-SMAC (IoT in Social, Mobile, Analytics and Cloud)(I-SMAC) (pp. 218-222). IEEE.

[10] Petkovski, A. J., Stojkoska, B. L. R., Trivodaliev, K. V., \& Kalajdziski, S. A. (2016, November). Analysis of churn prediction: A case study on telecommunication services in Macedonia. In 2016 24th Telecommunications Forum (TELFOR) (pp. 1-4). IEEE.

[11] Vijaya, J., \& Sivasankar, E. (2019). An efficient system for customer churn prediction through particle swarm optimization based feature selection model with simulated annealing. Cluster Computing, 22(5), 10757-10768.

[12] Amin, A., Al-Obeidat, F., Shah, B., Adnan, A., Loo, J., \& Anwar, S. (2019). Customer churn prediction in telecommunication industry using data certainty. Journal of Business Research, 94, 290-301. [13] Dalvi, P. K., Khandge, S. K., Deomore, A., Bankar, A., \& Kanade, V. A. (2016, March). Analyses of customer churn prediction in telecom industry using decision trees and logistic regression. In 2016 Symposium on Colossal Data Analysis and Networking (CDAN) (pp. 1-4). IEEE. [14] Pamina, J., Raja, B., SathyaBama, S., Sruthi, M. S., \& VJ, A. (2019). An effective classifier for predicting churn in telecommunication. Jour of Adv Research in Dynamical \& Control Systems, 11.

[15] Chen, C. P., Weng, J. Y., Yang, C. S., \& Tseng, F. M. (2018). Employing a data mining approach for identification of mobile opinion leaders and their content usage patterns in large telecommunications datasets. Technological Forecasting and Social Change, 130, 88-98. 
523

524

525

526

527

528

529

530

531

532

533

534

535

536

537

538

539

540

541

542

543

544

545

546

547

548

549

550

551

552

553

554

555

556

557

558

559

560

561

562

563

564

565

566

[16] Halibas, A. S., Matthew, A. C., Pillai, I. G., Reazol, J. H., Delvo, E. G., \& Reazol, L. B. (2019, January). Determining the intervening effects of exploratory data analysis and feature engineering in telecoms customer churn modelling. In 2019 4th MEC International Conference on Big Data and Smart City (ICBDSC) (pp. 1-7). IEEE.

[17] Nijhawan, V. K., Madan, M., \& Dave, M. (2019). An Analytical Implementation of CART Using RStudio for Churn Prediction. In Information and Communication Technology for Competitive Strategies (pp. 109-120). Springer, Singapore.

[18] Kumar, S., \& Kumar, M. (2019, May). Predicting Customer Churn Using Artificial Neural Network. In International Conference on Engineering Applications of Neural Networks (pp. 299306). Springer, Cham.

[19] Zhong, J., \& Li, W. (2019, March). Predicting customer churn in the telecommunication industry by analyzing phone call transcripts with convolutional neural networks. In Proceedings of the 2019 3rd International Conference on Innovation in Artificial Intelligence (pp. 55-59). [20] Amin, A., Shah, B., Abbas, A., Anwar, S., Alfandi, O., \& Moreira, F. (2019, April). Features Weight estimation using a genetic algorithm for customer churn prediction in the telecom sector. In World conference on information systems and technologies (pp. 483-491). Springer, Cham.

[21] Babaiyan, V., \& Sarfarazi, S. A. (2019). Analyzing customers of South Khorasan telecommunication company with expansion of RFM to LRFM model. Journal of AI and Data Mining, 7(2), 331-340.

[22] Amin, A., Al-Obeidat, F., Shah, B., Adnan, A., Loo, J., \& Anwar, S. (2019). Customer churn prediction in telecommunication industry using data certainty. Journal of Business Research, 94, 290-301.

[23] Saghir, M., Bibi, Z., Bashir, S., \& Khan, F. H. (2019, January). Churn prediction using neural network based individual and ensemble models. In 2019 16th International Bhurban Conference on Applied Sciences and Technology (IBCAST) (pp. 634-639). IEEE. [24] Ullah, I., Raza, B., Malik, A. K., Imran, M., Islam, S. U., \& Kim, S. W. (2019). A Churn Prediction Model Using Random Forest: Analysis of Machine Learning Techniques for Churn Prediction and Factor Identification in Telecom Sector. IEEE Access, 7, 60134-60149. [25] Arafat, M., Qusef, A., \& Sammour, G. (2019, April). Detection of Wangiri Telecommunication Fraud Using Ensemble Learning. In 2019 IEEE Jordan International Joint Conference on Electrical Engineering and Information Technology (JEEIT)(pp. 330-335). IEEE. [26] Bharat, A. (2019). Consumer Engagement Pattern Analysis Leading to Improved Churn Analytics: An Approach for Telecom Industry. In Data Management, Analytics and Innovation (pp. 203-211). Springer, Singapore.

[27] Gajowniczek, K., Orłowski, A., \& Ząbkowski, T. (2019). Insolvency modeling with generalized entropy cost function in neural networks. Physica A: Statistical Mechanics and its Applications, 526, 120730.

[28] Ahmed, M., Afzal, H., Siddiqi, I., Amjad, M. F., \& Khurshid, K. (2020). Exploring nested ensemble learners using overproduction and choose approach for churn prediction in telecom industry. Neural Computing and Applications, 32(8), 3237-3251.

[29] Brownlow, J., Chu, C., Fu, B., Xu, G., Culbert, B., \& Meng, Q. (2018, May). Cost-sensitive churn prediction in fund management services. In International Conference on Database Systems for Advanced Applications (pp. 776-788). Springer, Cham.

Peer] Comput. Sci. reviewing PDF | (CS-2021:07:64230:1:1:NEW 1 Dec 2021) 
567 [30] Vo, N. N., Liu, S., Brownlow, J., Chu, C., Culbert, B., \& Xu, G. (2018, May). Client Churn

568 Prediction with Call Log Analysis. In International Conference on Database Systems for

569 Advanced Applications (pp. 752-763). Springer, Cham.

570 [31] Calzada-Infante, L., Óskarsdóttir, M., \& Baesens, B. (2020). Evaluation of customer

571 behavior with temporal centrality metrics for churn prediction of prepaid contracts. Expert

572 Systems with Applications, 160, 113553.

573 [32] Nguyen, N. N., \& Duong, A. T. (2021). Comparison of Two Main Approaches for Handling

574 Imbalanced Data in Churn Prediction Problem. Journal of Advances in Information Technology

575 Vol, 12(1).

576 [33] Vural, U., Okay, M. E., \& Yildiz, E. M. (2020). Churn Prediction for Telecommunication

577 Industry Using Artificial Neural Networks. International Journal of Computer and Information

578 Engineering, 14(11), 396-399.

579 [34] Jain, H., Khunteta, A., \& Srivastava, S. (2020). Telecom churn prediction and used

580 techniques, datasets and performance measures: a review. Telecommunication Systems, 1-18.

581 [35] Maldonado, S., Domínguez, G., Olaya, D., \& Verbeke, W. (2021). Profit-driven churn

582 prediction for the mutual fund industry: A multisegment approach. Omega, 100, 102380.

583 [36] Azeem, M., Usman, M., \& Fong, A. C. M. (2017). A churn prediction model for prepaid

584 customers in telecom using fuzzy classifiers. Telecommunication Systems, 66(4), 603-614.

585 [37] Krawczyk, B., Minku, L. L., Gama, J., Stefanowski, J., \& Woźniak, M. (2017). Ensemble

586 learning for data stream analysis: A survey. Information Fusion, 37, 132-156.

587 [38] Hammoudeh, A., Fraihat, M., \& Almomani, M. (2019, April). Selective Ensemble Model

588 for Telecom Churn Prediction. In 2019 IEEE Jordan International Joint Conference on Electrical

589 Engineering and Information Technology (JEEIT) (pp. 485-487). IEEE.

590 [39] Amin, A., Shah, B., Khattak, A. M., Baker, T., \& Anwar, S. (2018, July). Just-in-time

591 customer churn prediction: With and without data transformation. In 2018 IEEE congress on

592 evolutionary computation (CEC) (pp. 1-6). IEEE.

593 [40] Chen, W. (2017, December). Customer Churn Analysis for Telecom Operators Based on

594 SVM. In Signal and Information Processing, Networking and Computers: Proceedings of the 3rd

595 International Conference on Signal and Information Processing, Networking and Computers

596 (ICSINC) (Vol. 473, p. 327). Springer.

597 [41] Zhang, X., Zhang, Z., Liang, D., \& Jin, H. (2018, August). A Novel Decision Tree Based on

598 Profit Variance Maximization Criterion for Customer Churn Problem. In 2018 10th International

599 Conference on Intelligent Human-Machine Systems and Cybernetics (IHMSC) (Vol. 1, pp. 20-

600 23). IEEE.

601 [42] Zhu, B., Xie, G., Yuan, Y., \& Duan, Y. (2018, May). Investigating Decision Tree in Churn

602 Prediction with Class Imbalance. In Proceedings of the International Conference on Data

603 Processing and Applications (pp. 11-15).

604 [43] De Caigny, A., Coussement, K., \& De Bock, K. W. (2018). A new hybrid classification

605 algorithm for customer churn prediction based on logistic regression and decision

606 trees. European Journal of Operational Research, 269(2), 760-772.

607 [44] Azeem, M., \& Usman, M. (2018). A fuzzy based churn prediction and retention model for

608 prepaid customers in telecom industry. International Journal of Computational Intelligence

609 Systems, 11(1), 66-78.

610 [45] Azeem, M., Usman, M., \& Fong, A. C. M. (2017). A churn prediction model for prepaid

611 customers in telecom using fuzzy classifiers. Telecommunication Systems, 66(4), 603-614.

Peer] Comput. Sci. reviewing PDF | (CS-2021:07:64230:1:1:NEW 1 Dec 2021) 
612 [46] Amin, A., Shah, B., Khattak, A. M., Baker, T., \& Anwar, S. (2018, July). Just-in-time 613 customer churn prediction: With and without data transformation. In 2018 IEEE congress on 614 evolutionary computation (CEC) (pp. 1-6). IEEE.

615 [47] Stripling, E., vanden Broucke, S., Antonio, K., Baesens, B., \& Snoeck, M. (2018). Profit 616 maximizing logistic model for customer churn prediction using genetic algorithms. Swarm and 617 Evolutionary Computation, 40, 116-130.

618 [48] Zhu, B., Baesens, B., Backiel, A., \& Vanden Broucke, S. K. (2018). Benchmarking 619 sampling techniques for imbalance learning in churn prediction. Journal of the Operational 620 Research Society, 69(1), 49-65.

621 [49] Mahajan, R., \& Som, S. (2016, March). Customer behavior patterns analysis in Indian 622 mobile telecommunications industry. In 2016 3rd International Conference on Computing for 623 Sustainable Global Development (INDIACom) (pp. 1165-1169). IEEE.

624 [50] Mishra, A., \& Reddy, U. S. (2017, November). A comparative study of customer churn

625 prediction in telecom industry using ensemble based classifiers. In 2017 International

626 Conference on Inventive Computing and Informatics (ICICI) (pp. 721-725). IEEE.

627 [51] Ahmed, A. A., \& Maheswari, D. (2017). Churn prediction on huge telecom data using 628 hybrid firefly based classification. Egyptian Informatics Journal, 18(3), 215-220.

629 [52] Yu, R., An, X., Jin, B., Shi, J., Move, O. A., \& Liu, Y. (2018). Particle classification 630 optimization-based BP network for telecommunication customer churn prediction. Neural 631 Computing and Applications, 29(3), 707-720.

632 [53] Khairandish, M. O., Sharma, M., Jain, V., Chatterjee, J. M., \& Jhanjhi, N. Z. (2021). A

633

634

635

636

637

638

639

640

641

642

643

644

645

646

647

648

649

650

651

652

653

654

655

656

657

658

659
Hybrid CNN-SVM Threshold Segmentation Approach for Tumor Detection and Classification of MRI Brain Images. IRBM.

[54] SL, A., Chatterjee, J. M., Alaboudi, A., \& Jhanjhi, N. Z. (2021). A Machine Learning Way

to Classify Autism Spectrum Disorder. International Journal of Emerging Technologies in

Learning, 16(6)

[55] Gupta, M. K., \& Chandra, P. (2020). A comprehensive survey of data mining. International Journal of Information Technology, 12(4), 1243-1257.

[56] G. Brown, J. Wyatt, R. Harris, X. Yao, "Diversity Creation Methods: A Survey and

Categorisation," Journal of Information Fusion (Special issue on Diversity in Multiple Classifier

Systems). Volume 6, issue 1, pp 5-20, March 2005

[57] Adhikary, D. D., \& Gupta, D. (2020). Applying over 100 classifiers for churn prediction in telecom companies. Multimedia Tools and Applications, 1-22.

[58] Saghir, M., Bibi, Z., Bashir, S., \& Khan, F. H. (2019, January). Churn prediction using neural network based individual and ensemble models. In 2019 16th International Bhurban Conference on Applied Sciences and Technology (IBCAST) (pp. 634-639). IEEE.

[59] Singh, B. E. R., \& Sivasankar, E. (2019). Enhancing Prediction Accuracy of Default of Credit Using Ensemble Techniques. In First International Conference on Artificial Intelligence and Cognitive Computing (pp. 427-436). Springer, Singapore.

[6o] Ullah, I., Raza, B., Malik, A. K., Imran, M., Islam, S. U., \& Kim, S. W. (2019). A churn prediction model using random forest: analysis of machine learning techniques for churn prediction and factor identification in telecom sector. IEEE Access, 7, 60134-60149.

[61] Rupapara, V., Rustam, F., Shahzad, H. F., Mehmood, A., Ashraf, I., \& Choi, G. S. (2021). Impact of SMOTE on Imbalanced Text Features for Toxic Comments Classification using RVVC Model. IEEE Access.

[62] Jamil, R., Ashraf, I., Rustam, F., Saad, E., Mehmood, A., \& Choi, G. S. (2021). Detecting sarcasm in multi-domain datasets using convolutional neural networks and long short term memory network model. PeerJ Computer Science, 7, e645. 
660 [63] Rustam, F., Ashraf, I., Mehmood, A., Ullah, S., \& Choi, G. S. (2019). Tweets classification on 661 the base of sentiments for US airline companies. Entropy, 21(11), 1078.

662 [64] Rustam, F., Mehmood, A., Ullah, S., Ahmad, M., Khan, D. M., Choi, G. S., \& On, B. W. 663 (2020). Predicting pulsar stars using a random tree boosting voting classifier (RTB-

664 VC). Astronomy and Computing, 32, 100404.

665 [65] Rustam, F., Khalid, M., Aslam, W., Rupapara, V., Mehmood, A., \& Choi, G. S. (2021). A 666 performance comparison of supervised machine learning models for Covid-19 tweets sentiment 667 analysis. Plos one, 16(2), e0245909.

668 [66] Omar, B., Rustam, F., Mehmood, A., \& Choi, G. S. (2021). Minimizing the overlapping 669 degree to improve class-imbalanced learning under sparse feature selection: application to fraud 670 detection. IEEE Access, 9, 28101-28110. 
Figure 1

Proposed customer churn prediction model 


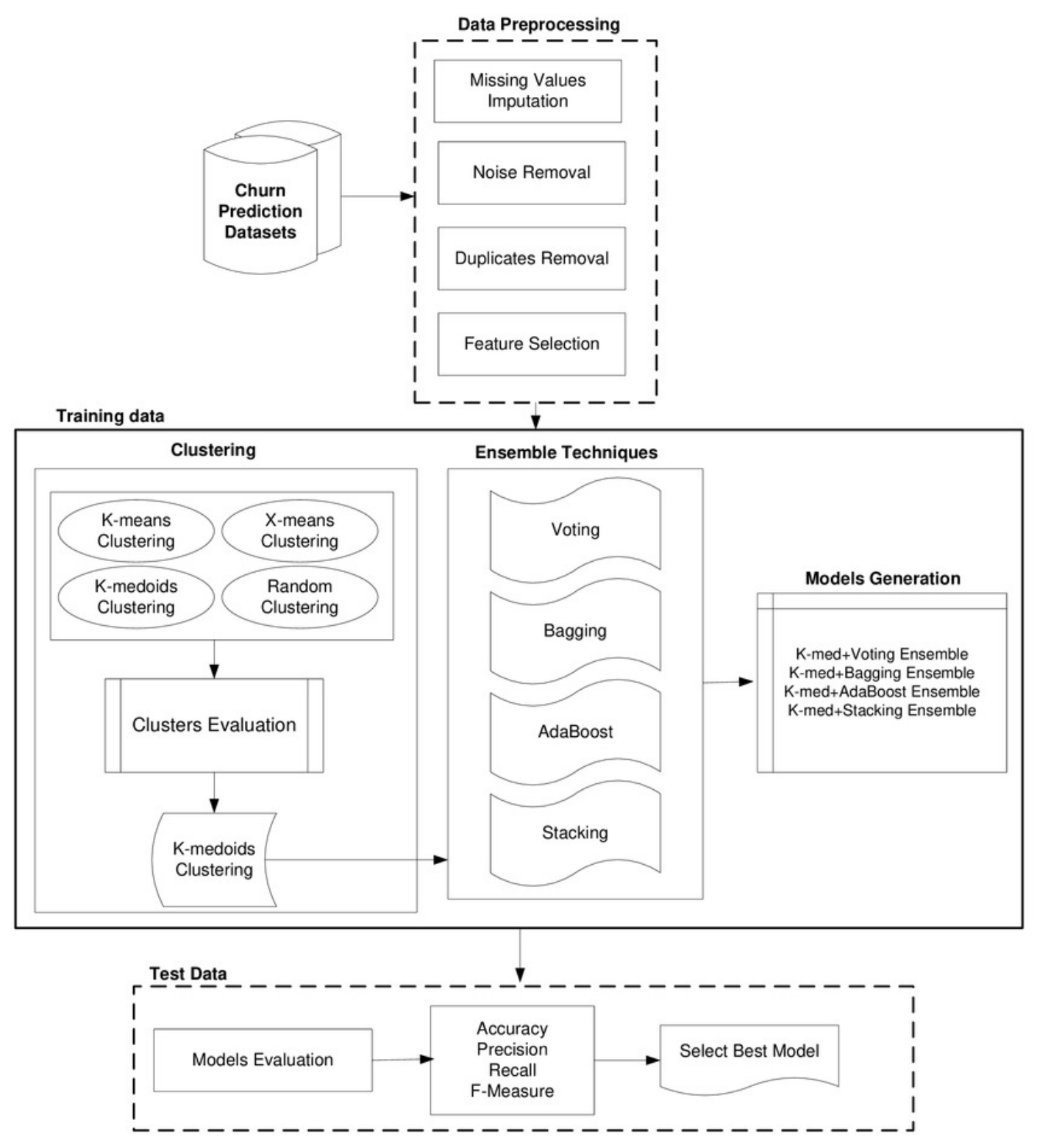




\section{Table $\mathbf{1}$ (on next page)}

State of the Art Techniques for Customer Churn Prediction 
1

2
Table 1: State of the art techniques for customer churn prediction

\begin{tabular}{|c|c|c|c|c|c|c|c|}
\hline Ref & Year & Author & Techniques & Accuracy & Precision & Recall & F-Measure \\
\hline \multirow[t]{4}{*}[35]{} & \multirow[t]{4}{*}{2021} & \multirow{4}{*}{$\begin{array}{c}\text { Maldonado, S., } \\
\text { Domínguez, G., Olaya, } \\
\text { D., \& Verbeke, W. }\end{array}$} & Logit & $56.43 \%$ & - & - & - \\
\hline & & & K Nearest Neighbor & $64.07 \%$ & - & - & - \\
\hline & & & CART & $57.05 \%$ & - & - & - \\
\hline & & & Random Forest & $66.24 \%$ & - & - & - \\
\hline \multirow[t]{4}{*}[57]{} & \multirow[t]{4}{*}{$\underline{2020}$} & \multirow{4}{*}{$\frac{\text { Adhikary, D. D., \& }}{\text { Gupta, D. }}$} & Voting & $71.3 \%$ & NA & $71.4 \%$ & $\mathrm{NA}$ \\
\hline & & & Bagging & $71.9 \%$ & $69.3 \%$ & $71.9 \%$ & $\underline{62.1 \%}$ \\
\hline & & & AdaBoost & $71.3 \%$ & $\mathrm{NA}$ & $71.4 \%$ & NA \\
\hline & & & Stacking & $71.3 \%$ & $\mathrm{NA}$ & $71.4 \%$ & $\mathrm{NA}$ \\
\hline$[33]$ & 2020 & $\begin{array}{c}\text { Vural, U., Okay, M. E., } \\
\text { \& Yildiz, E. M } \\
\end{array}$ & Artificial Neural Network & $89 \%$ & - & - & - \\
\hline \multirow[t]{2}{*}[58]{} & \multirow[t]{2}{*}{2019} & \multirow{2}{*}{$\begin{array}{l}\frac{\text { Saghir, M., Bibi, Z., }}{\text { Bashir, S., \& Khan, F. }} \\
\text { H. }\end{array}$} & Bagging & $\underline{80.8 \%}$ & $\underline{81.88 \%}$ & $75.28 \%$ & $78.44 \%$ \\
\hline & & & AdaBoost & $73.9 \%$ & $70.46 \%$ & $\underline{73.74 \%}$ & $72.06 \%$ \\
\hline \multirow[t]{2}{*}{ [59] } & \multirow[t]{2}{*}{$\underline{2019}$} & \multirow{2}{*}{$\begin{array}{l}\text { Singh, B. E. R., \& } \\
\text { Sivasankar, E. }\end{array}$} & Bagging & $79.13 \%$ & NA & NA & NA \\
\hline & & & Boosting & $82.03 \%$ & $\underline{\mathrm{NA}}$ & NA & NA \\
\hline \multirow[t]{3}{*}[14]{} & \multirow[t]{3}{*}{2019} & \multirow{3}{*}{$\begin{array}{c}\text { Pamina, J., Raja, B., } \\
\text { SathyaBama }\end{array}$} & $\mathrm{XG}$ & $79.8 \%$ & - & - & $58.2 \%$ \\
\hline & & & K nearest neighbor & $75.4 \%$ & - & - & $49 \%$ \\
\hline & & & Random Forest & $77 \%$ & - & - & $50.6 \%$ \\
\hline$[16]$ & 2019 & Halibas, A. S., Matthew & Gradient Boosted Tree & $79.1 \%$ & $73.1 \%$ & $79.6 \%$ & $76.2 \%$ \\
\hline$[20]$ & 2019 & $\begin{array}{c}\text { Amin, A., Shah, B., } \\
\text { Abbas, }\end{array}$ & $\begin{array}{l}\text { Genetic Algorithm +Naïve } \\
\text { Bayes }\end{array}$ & $89.1 \%$ & $95.65 \%$ & $16.92 \%$ & $28.76 \%$ \\
\hline$[23]$ & 2019 & $\begin{array}{l}\text { ] Saghir, M., Bibi, Z., } \\
\text { Bashir } \\
\end{array}$ & $\begin{array}{c}\text { Ensemble Classifier with } \\
\text { Bagging and Neural network }\end{array}$ & $81 \%$ & 81.56 & 73.74 & 72.06 \\
\hline \multirow[t]{2}{*}[24]{} & \multirow[t]{2}{*}{2019} & \multirow[t]{2}{*}{$\begin{array}{l}\text { Ullah, I., Raza, B., } \\
\text { Malik, A. K }\end{array}$} & Random Forest & $88 \%$ & $89.1 \%$ & $89.6 \%$ & $87.6 \%$ \\
\hline & & & Random tree & $.85 \%$ & - & - & $21.5 \%$ \\
\hline$[26]$ & 2019 & Bharat, A & Logistic Regression & $70 \%$ & - & - & - \\
\hline$[27]$ & 2019 & $\begin{array}{l}\text { Gajowniczek, K., } \\
\text { Orłowski, A. }\end{array}$ & $\begin{array}{c}\text { Entropy Cost Function Neural } \\
\text { network }\end{array}$ & $60 \%$ & $74 \%$ & $77 \%$ & N/A \\
\hline \multirow[t]{4}{*}{ [3] } & \multirow[t]{4}{*}{2018} & \multirow{4}{*}{$\begin{array}{l}\text { J. Vijaya and E. } \\
\text { Sivasankar }\end{array}$} & K-means & $87.61 \%$ & $93.68 \%$ & $12.23 \%$ & - \\
\hline & & & K-mediods & $90.91 \%$ & $98 \%$ & $28.4 \%$ & - \\
\hline & & & Naïve Bayes & $25.5 \%$ & $100 \%$ & -- & - \\
\hline & & & K Nearest Neighbor & $91.39 \%$ & $99 \%$ & $01 \%$ & - \\
\hline [4] & 2018 & $\begin{array}{c}\text { Höppner, S., Stripling, } \\
\text { E }\end{array}$ & EMPC with Decision Tree & $89 \%$ & $94.81 \%$ & - & $60.7 \%$ \\
\hline \multirow[t]{3}{*}[5]{} & \multirow[t]{3}{*}{2018} & \multirow[t]{3}{*}{ Ali, M., Rehman, A. } & Support Vector Machine & $90 \%$ & $98.2 \%$ & $\mathrm{~N} / \mathrm{A}$ & $98.1 \%$ \\
\hline & & & Bagging Stacking & $85.5 \%$ & $73.1 \%$ & & $78.8 \%$ \\
\hline & & & Naïve Bayes & $92.9 \%$ & $92.7 \%$ & & $92.7 \%$ \\
\hline \multirow[t]{4}{*}[6]{} & \multirow[t]{4}{*}{2018} & \multirow{4}{*}{$\begin{array}{c}\text { Amin, A., Shah, B., } \\
\text { Khattak }\end{array}$} & Naïve Bayes & $86 \%$ & $\mathrm{~N} / \mathrm{A}$ & $\mathrm{N} / \mathrm{A}$ & $16.7 \%$ \\
\hline & & & K Nearest Neighbor & $85 \%$ & - & - & $16.6 \%$ \\
\hline & & & Gradient Boosted Tree & $72 \%$ & - & - & $17.3 \%$ \\
\hline & & & SRI & $16.7 \%$ & - & - & $87 \%$ \\
\hline
\end{tabular}




\begin{tabular}{|c|c|c|c|c|c|c|c|}
\hline & & & $\mathrm{DP}$ & $80 \%$ & - & - & $16.0 \%$ \\
\hline$[39]$ & 2018 & Amin, A., Shah, B & JIT & $59 \%$ & - & - & - \\
\hline$[40]$ & 2018 & $\begin{array}{c}\text { Runsha Dong }(\&), \text { Fei } \\
\text { Su, }\end{array}$ & Support Vector Machine & $70.6 \%$ & - & - & - \\
\hline$[30]$ & 2018 & $\begin{array}{l}\text { Vo, N. N., Liu, S., } \\
\text { Brownlo }\end{array}$ & XGBoostAlgorithm & $81.08 \%$ & - & - & - \\
\hline$[41]$ & 2018 & $\begin{array}{c}\text { Zhang, X., Zhang, Z., } \\
\text { Liang }\end{array}$ & Decision Tree & $70 \%$ & - & - & - \\
\hline$[42]$ & 2018 & $\begin{array}{c}\text { Zhu, B., Xie, G., Yuan, } \\
\text { Y }\end{array}$ & CART & $67.97 \%$ & - & - & $100 \%$ \\
\hline \multirow[t]{2}{*}[43]{} & \multirow[t]{2}{*}{2018} & \multirow{2}{*}{$\begin{array}{l}\text { De Caigny, A., } \\
\text { Coussement, K }\end{array}$} & Logistic regression & $88.12 \%$ & - & - & - \\
\hline & & & Decision Tree & $88 \%$ & - & - & - \\
\hline$[12]$ & 2017 & Amin, A., Al-Obeidat & Naïve Bayes & $57 \%$ & $54.14 \%$ & $61.30 \%$ & $57.50 \%$ \\
\hline$[46]$ & 2017 & $\begin{array}{c}\text { Amin, A., Al-Obeidat, } \\
\text { F., Shah }\end{array}$ & JIT & $55.3 \%$ & $57.62 \%$ & $40.05 \%$ & $47.26 \%$ \\
\hline$[47]$ & 2017 & $\begin{array}{l}\text { Stripling, E., vanden } \\
\text { Broucke, S } \\
\end{array}$ & Proflogit & $70 \%$ & - & - & - \\
\hline$[48]$ & 2017 & $\begin{array}{l}\text { Zhu, B., Baesens, B., } \\
\text { Backiel, A }\end{array}$ & Sampling Method & $86.06 \%$ & - & - & - \\
\hline [49] & 2017 & Mahajan, R., \& Som, S & $\begin{array}{c}\text { K-Local Maximum Features } \\
\text { Extraction Method }\end{array}$ & $80 \%$ & $50 \%$ & $22 \%$ & $94 \%$ \\
\hline \multirow[t]{4}{*}[50]{} & \multirow[t]{4}{*}{2017} & \multirow[t]{4}{*}{ Mishra, A., \& Reddy } & Bagging & $90.83 \%$ & - & $92.02 \%$ & - \\
\hline & & & Boosting & $90.32 \%$ & & $97.91 \%$ & - \\
\hline & & & Random Forest & $91.67 \%$ & $83.11 \%$ & $98.89 \%$ & - \\
\hline & & & Decision Tree & $90.96 \%$ & - & - & - \\
\hline [9] & 2016 & $\begin{array}{l}\text { Tiwari, A., Sam, R., \& } \\
\text { Shaikh, }\end{array}$ & Naïve Bayes & $70 \%$ & - & - & - \\
\hline \multirow[t]{3}{*}[10]{} & \multirow[t]{2}{*}{2016} & \multirow{2}{*}{$\begin{array}{l}\text { Petkovski, A. J., } \\
\text { Stojkoska }\end{array}$} & Naïve Bayes & $85.24 \%$ & - & & $82 \%$ \\
\hline & & & $\mathrm{C} 4.5$ & $91.57 \%$ & & & $84 \%$ \\
\hline & & & K Nearest Neighbor & $90.59 \%$ & & & $85 \%$ \\
\hline$[51]$ & 2016 & $\begin{array}{c}\text { Ahmed, A. A., \& } \\
\text { Maheswari }\end{array}$ & Firefly Algorithm & $86.38 \%$ & $90 \%$ & $80 \%$ & $93 \%$ \\
\hline$[52]$ & 2016 & $\begin{array}{l}\text { Yu,R ., An, X., Jin, B., } \\
\text { Shi, J., }\end{array}$ & $\begin{array}{l}\text { Particle Classification } \\
\text { Optimization Based BP } \\
\end{array}$ & $69.64 \%$ & $87.84 \%$ & $51.43 \%$ & $48.57 \%$ \\
\hline
\end{tabular}

3 


\section{Table 2 (on next page)}

Clustering Evaluation on Churn Prediction Datasets 
1

2

\begin{tabular}{|c|c|c|c|c|c|c|c|}
\hline Technique & Accuracy & Recall & Precision & F-measure & RMSE & MSE & MAE \\
\hline \multicolumn{8}{|c|}{ Clustering Evaluation on GitHub churn prediction dataset } \\
\hline X-means & 50.58 & 52.05 & 14.72 & 22.94 & 0.78 & .6084 & 0.15 \\
\hline K-means & 50.58 & 52.05 & 14.72 & 22.94 & 0.78 & .6084 & 0.15 \\
\hline K-med & 65.44 & 29.13 & 14.37 & 19.25 & 0.69 & 0.4761 & 0.11 \\
\hline Random & 50.96 & 48.93 & 14.19 & 22.01 & 0.75 & .5625 & 0.14 \\
\hline \multicolumn{8}{|c|}{ Clustering Evaluation on Bigml churn prediction dataset } \\
\hline X-means & 50.04 & 48.86 & 14.26 & 22.08 & 0.63 & 0.3969 & .0992 \\
\hline K-means & 50.04 & 48.86 & 14.26 & 22.08 & 0.63 & 0.3969 & .0992 \\
\hline K-med & 55.56 & 41.82 & 14.40 & 21.43 & 0.54 & 0.2916 & .0729 \\
\hline Random & 50.94 & 49.06 & 14.57 & 22.47 & 0.61 & 0.3721 & 0.093 \\
\hline
\end{tabular}

3 


\section{Table 3 (on next page)}

Clustering with Single Classifier on Churn Prediction Datasets 
Table 3: Clustering with Single Classifier on Churn Prediction datasets

\begin{tabular}{|c|c|c|c|c|c|c|c|c|}
\hline Technique & Accuracy & Recall & Precision & F-measure & Accuracy & Recall & Precision & F-measure \\
\hline & \multicolumn{6}{|c|}{ GitHub dataset } \\
\hline K med+GBT & $\mathbf{9 4}$ & $\mathbf{6 2 . 2 3}$ & $\mathbf{9 3 . 0 2}$ & $\mathbf{7 4 . 5 7}$ & $\mathbf{9 2 . 2 5}$ & $\mathbf{5 1 . 9 6}$ & $\mathbf{9 0 . 6 1}$ & $\mathbf{6 6 . 0 5}$ \\
\hline K med+DT & 86.4 & 4.10 & 93.54 & 7.85 & 86.76 & 8.90 & 97.72 & 16.31 \\
\hline K med+RF & 87.6 & 14.14 & 88.49 & 24.39 & 91.53 & 65.42 & 73.31 & 69.14 \\
\hline K med+KNN & 86.02 & 12.58 & 52.35 & 20.29 & 84.39 & 2.61 & 20.63 & 4.76 \\
\hline K med+DL & 92.5 & 68.45 & 76.10 & 72.07 & 91.53 & 65.42 & 73.31 & 69.14 \\
\hline K med+NB & 87.02 & 52.61 & 54.22 & 53.40 & 83.28 & 43.47 & 42.51 & 42.98 \\
\hline K med+NB(K) & 83.56 & 42.43 & 41.95 & 42.19 & 91.14 & 52.79 & 79.19 & 63.35 \\
\hline Average & 88.15 & 36.65 & 71.38 & 42.11 & 88.70 & 41.52 & 68.18 & 47.39 \\
\hline
\end{tabular}

3

4 


\section{Table 4 (on next page)}

Clustering with Voting Ensemble for Churn Prediction Datasets 
1

Table 4: Clustering with Voting Ensemble for Churn Prediction Datasets

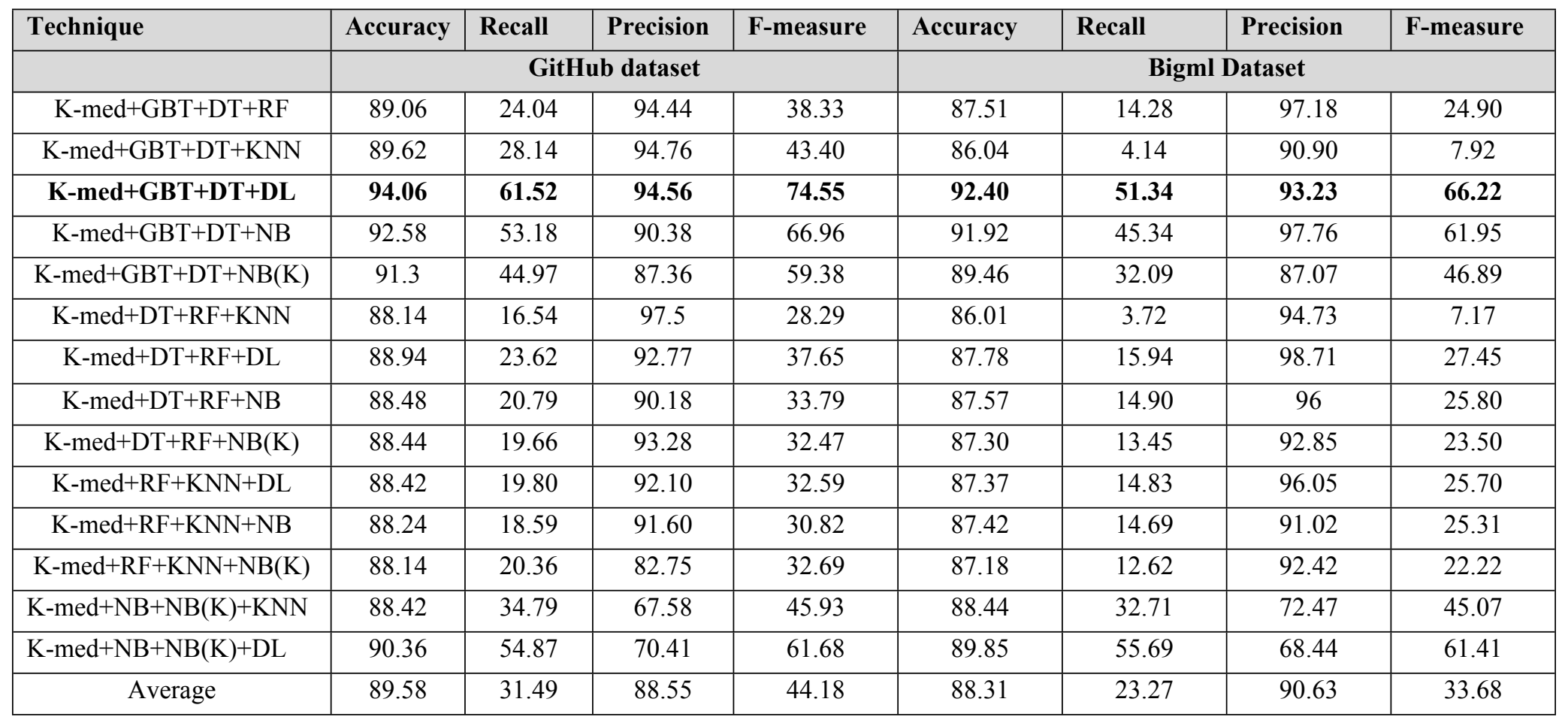

2 


\section{Table 5 (on next page)}

Clustering with Bagging Ensemble on Churn Prediction Datasets 
1

2

\begin{tabular}{|c|c|c|c|c|c|c|c|c|c|}
\hline Technique & Accuracy & Recall & Precision & F-measure & Accuracy & Recall & Precision & F-measure \\
\hline & \multicolumn{5}{|c|}{ GitHub Dataset } \\
\hline K-med+GBT+DT+RF & 89.12 & 24.46 & 94.53 & 38.87 & 87.54 & 14.49 & 97.22 & 25.22 \\
\hline K-med+GBT+DT+kNN & 89.7 & 28.71 & 94.85 & 44.08 & 86.10 & 4.55 & 91.66 & 8.67 \\
\hline K-med+GBT+DT+DL & $\mathbf{9 4 . 1 2}$ & $\mathbf{6 1 . 1 0}$ & $\mathbf{9 5 . 7 8}$ & $\mathbf{7 4 . 6 1}$ & $\mathbf{9 2 . 4 1}$ & $\mathbf{5 1 . 5 5}$ & $\mathbf{9 3 . 2 5}$ & $\mathbf{6 6 . 4}$ \\
\hline K-med+GBT+DT+NB & 92.64 & 53.60 & 90.45 & 67.31 & 91.98 & 45.75 & 97.78 & 62.34 \\
\hline K-med+GBT+DT+NB(K) & 91.4 & 45.68 & 87.53 & 60.03 & 89.52 & 32.50 & 87.22 & 47.36 \\
\hline K-med+DT+RF+kNN & 88.1 & 16.26 & 97.45 & 27.87 & 86.04 & 3.93 & 95 & 7.55 \\
\hline K-med+DT+RF+DL & 88.98 & 23.90 & 92.85 & 38.02 & 87.81 & 16.14 & 98.73 & 27.75 \\
\hline K-med+DT+RF+NB & 88.5 & 20.79 & 90.74 & 33.83 & 87.66 & 15.52 & 96.15 & 26.73 \\
\hline K-med+DT+RF+NB(K) & 88.42 & 19.23 & 94.44 & 31.96 & 87.33 & 13.66 & 92.95 & 23.82 \\
\hline K-med+RF+kNN+DL & 88.32 & 18.52 & 94.24 & 30.96 & 87.63 & 15.32 & 96.10 & 26.42 \\
\hline K-med+RF+kNN+NB & 88.04 & 16.83 & 92.24 & 28.46 & 87.45 & 14.90 & 91.13 & 25.62 \\
\hline K-med+RF+kNN+NB(K) & 88 & 17.11 & 89.62 & 28.74 & 87.24 & 13.04 & 92.64 & 22.86 \\
\hline K-med+NB+NB(K)+kNN & 88.66 & 33.94 & 70.58 & 45.84 & 88.47 & 32.91 & 72.60 & 45.29 \\
\hline K-med+NB+NB(K)+DL & 90.62 & 54.87 & 72.11 & 62.32 & 89.88 & 55.90 & 68.52 & 61.57 \\
\hline Average & 89.61 & 31.07 & 89.82 & 43.78 & 88.37 & 23.58 & 90.78 & 34.11 \\
\hline
\end{tabular}

Table 5: Clustering with Bagging ensemble on Churn Prediction datasets 


\section{Table 6 (on next page)}

Clustering with Stacking Ensemble on Churn Prediction Datasets 
1

2

\begin{tabular}{|c|c|c|c|c|c|c|c|c|c|c|}
\hline Technique & Accuracy & Recall & Precision & F-measure & Accuracy & Recall & Precision & F-measure \\
\hline & \multicolumn{5}{|c|}{ GitHub Dataset } \\
\hline K-med+GBT+DT+RF & 94.34 & 76.09 & 82.51 & 79.17 & 92.39 & 67.49 & 77.80 & 72.28 \\
\hline K-med+GBT+DT+KNN & 94.56 & 73.26 & 86.18 & 79.20 & 92.31 & 66.04 & 77.61 & 71.36 \\
\hline K-med+GBT+DT+DL & $\mathbf{9 4 . 6 5}$ & $\mathbf{7 3 . 1 2}$ & $\mathbf{8 7 . 3 3}$ & $\mathbf{7 9 . 5 9}$ & $\mathbf{9 2 . 4 0}$ & $\mathbf{6 6 . 2 5}$ & $\mathbf{7 8 . 0 4}$ & $\mathbf{7 1 . 6 6}$ \\
\hline K-med+GBT+DT+NB & 94.5 & 74.82 & 84.50 & 79.36 & 89.97 & 72.04 & 63.61 & 67.57 \\
\hline K-med+GBT+DT+NB(K) & 94.5 & 76.23 & 83.43 & 79.67 & 91.47 & 66.66 & 72.35 & 69.39 \\
\hline K-med+DT+RF+KNN & 86.02 & 12.58 & 52.35 & 20.29 & 87.12 & 13.66 & 84.61 & 23.52 \\
\hline K-med+DT+RF+DL & 92.38 & 69.02 & 75.07 & 71.92 & 91.32 & 68.73 & 70.63 & 69.67 \\
\hline K-med+DT+RF+NB & 86.86 & 49.50 & 53.84 & 51.58 & 91.50 & 44.72 & 93.10 & 60.41 \\
\hline K-med+DT+RF+NB(K) & 87.72 & 14.56 & 91.15 & 25.12 & 87.57 & 16.14 & 89.65 & 27.36 \\
\hline K-med+RF+KNN+DL & 92.2 & 67.04 & 75.11 & 70.85 & 91.05 & 64.18 & 71.26 & 67.53 \\
\hline K-med+RF+KNN+NB & 86.02 & 12.58 & 52.35 & 20.29 & 86.28 & 50.51 & 52.81 & 51.64 \\
\hline K-med+RF+KNN+NB(K) & 86.36 & 49.08 & 51.86 & 50.43 & 87.21 & 14.07 & 86.07 & 24.19 \\
\hline K-med+NB+NB(K)+KNN & 85.94 & 51.06 & 50.27 & 50.66 & 90.63 & 62.52 & 69.74 & 65.93 \\
\hline K-med+NB+NB(K)+DL & 92.96 & 69.73 & 78.12 & 73.69 & 86.82 & 55.48 & 54.47 & 54.97 \\
\hline Average & 90.66 & 55.05 & 71.70 & 59.49 & 89.87 & 52.05 & 74.42 & 56.97 \\
\hline
\end{tabular}

Table 6: Clustering with Stacking Ensemble on Churn Prediction datasets

3

4 


\section{Table 7 (on next page)}

Clustering with Stacking Ensemble on Churn Prediction Datasets 
1

Table 7: Clustering with AdaBoost Ensemble on Churn Prediction Datasets

2

\begin{tabular}{|c|c|c|c|c|c|c|c|c|}
\hline Technique & Accuracy & Recall & Precision & F-measure & Accuracy & Recall & Precision & F-measure \\
\hline & \multicolumn{4}{|c|}{ GitHub Dataset } & \multicolumn{4}{|c|}{ Bigml Dataset } \\
\hline $\mathrm{K}-\mathrm{med}+\mathrm{GBT}+\mathrm{DT}+\mathrm{RF}$ & 87.7 & 13.86 & 94.23 & 24.16 & 88.83 & 25.46 & 91.11 & 39.80 \\
\hline $\mathrm{K}-\mathrm{med}+\mathrm{GBT}+\mathrm{DT}+\mathrm{KNN}$ & 87.74 & 13.57 & 97.95 & 23.85 & 87.69 & 19.04 & 82.88 & 30.97 \\
\hline K-med+GBT+DT+DL & 94.7 & 75.10 & 87.04 & 80.63 & 92.43 & 66.45 & 78.10 & 71.81 \\
\hline $\mathrm{K}-\mathrm{med}+\mathrm{GBT}+\mathrm{DT}+\mathrm{NB}$ & 94.48 & 72.70 & 86.09 & 78.83 & 90.03 & 51.13 & 72.01 & 59.80 \\
\hline $\mathrm{K}$-med+GBT+DT+NB(K) & 93 & 59.97 & 86.35 & 70.78 & 88.23 & 39.95 & 65.42 & 49.61 \\
\hline $\mathrm{K}-\mathrm{med}+\mathrm{DT}+\mathrm{RF}+\mathrm{KNN}$ & 87.02 & 8.48 & 96.77 & 15.60 & 87.57 & 15.52 & 92.59 & 26.59 \\
\hline $\mathrm{K}-\mathrm{med}+\mathrm{DT}+\mathrm{RF}+\mathrm{DL}$ & 87.72 & 13.57 & 96.96 & 23.82 & 89.07 & 27.32 & 91.03 & 42.03 \\
\hline $\mathrm{K}-\mathrm{med}+\mathrm{DT}+\mathrm{RF}+\mathrm{NB}$ & 87.64 & 13.71 & 92.38 & 23.89 & 88.32 & 24.63 & 82.63 & 37.95 \\
\hline $\mathrm{K}-\mathrm{med}+\mathrm{DT}+\mathrm{RF}+\mathrm{NB}(\mathrm{K})$ & 87.52 & 13.29 & 89.52 & 23.15 & 88.17 & 19.95 & 91.42 & 32.76 \\
\hline $\mathrm{K}$-med+RF+KNN+DL & 88.22 & 19.09 & 88.81 & 31.43 & 87.75 & 16.56 & 94.11 & 28.16 \\
\hline $\mathrm{K}$-med+RF+KNN+NB & 88.08 & 17.96 & 88.81 & 29.88 & 87.54 & 15.52 & 91.46 & 26.54 \\
\hline $\mathrm{K}$-med+RF+KNN+NB(K) & 87.7 & 17.68 & 79.11 & 28.90 & 87.30 & 14.28 & 88.46 & 24.59 \\
\hline $\mathrm{K}-\mathrm{med}+\mathrm{NB}+\mathrm{NB}(\mathrm{K})+\mathrm{KNN}$ & 88.14 & 36.06 & 64.39 & 46.23 & 87.24 & 36.02 & 60 & 45.01 \\
\hline $\mathrm{K}-\mathrm{med}+\mathrm{NB}+\mathrm{NB}(\mathrm{K})+\mathrm{DL}$ & 90.12 & 58.41 & 67.37 & 62.57 & 89.31 & 55.90 & 65.37 & 60.26 \\
\hline Average & 89.29 & 30.82 & 87.66 & 40.24 & 88.54 & 30.54 & 81.89 & 41.13 \\
\hline
\end{tabular}

3

4 


\section{Table 8 (on next page)}

Average Accuracy Comparison of Different Techniques 


\begin{tabular}{|c|c|c|c|c|}
\hline Technique & Accuracy & Precision & Recall & F-Measure \\
\hline & \multicolumn{4}{|c|}{ GitHub dataset } \\
\hline Clustering & 54.39 & 45.54 & 14.50 & 22 \\
\hline Classification(Single Classifier) & 88.14 & 36.75 & 71.24 & 48.49 \\
\hline Clustering with Single Classifier & 88.15 & 36.65 & 71.38 & 42.11 \\
\hline Clustering with Voting Ensemble Classifier & 89.58 & 31.49 & 88.55 & 44.18 \\
\hline Clustering with Bagging Ensemble Classifier & 89.61 & 31.07 & 89.82 & 43.78 \\
\hline Clustering with Stacking Ensemble Classifier & 89.29 & 30.82 & 87.66 & 40.24 \\
\hline \multirow[t]{2}{*}{ Clustering with AdaBoost Ensemble Classifier } & 90.66 & 55.05 & 71.70 & 59.49 \\
\hline & \multicolumn{4}{|c|}{ Bigml dataset } \\
\hline Clustering & 51.65 & 47.15 & 14.38 & 22.03 \\
\hline Classification(Single Classifier) & 87.30 & 33.56 & 65.84 & 44.46 \\
\hline Clustering with Single Classifier & 88.70 & 41.52 & 68.18 & 47.39 \\
\hline Clustering with Voting Ensemble Classifier & 88.31 & 23.27 & 90.63 & 33.68 \\
\hline Clustering with Bagging Ensemble Classifier & 88.37 & 23.58 & 90.78 & 34.11 \\
\hline Clustering with Stacking Ensemble Classifier & 88.54 & 30.54 & 81.89 & 41.13 \\
\hline Clustering with AdaBoost Ensemble Classifier & 89.87 & 52.05 & 74.42 & 56.97 \\
\hline
\end{tabular}

3 


\section{Table 9 (on next page)}

Comparison with State of the Art techniques with Bigml Dataset 
1

Table 9: Comparison with State of the Art techniques with Bigml Dataset

\begin{tabular}{|c|c|c|c|c|c|c|c|}
\hline & Techniques & Reference & Accuracy & Precision & Recall & F-Measure & Standard Dev \\
\hline & JIT & [50] & 77.27 & 96.02 & 57.25 & 71.42 & NA \\
\hline & UDT & [20] & 84.0 & 52.38 & 64.71 & 57.89 & NA \\
\hline Ё & Multilayer Perception & [52] & 89.29 & 86.8 & 89.5 & 88.8 & NA \\
\hline 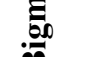 & Random Forest & [9] & 89.59 & 89.1 & 89.6 & 87.6 & NA \\
\hline 至 & Bagging + Deep Learning & [35] & 91.51 & 90.67 & 72.94 & 80.84 & NA \\
\hline$\frac{\pi}{8}$ & EWD & [64] & 88 & 86.01 & 78 & 79.01 & NA \\
\hline 6 & $\mathrm{NB}+\mathrm{LR}$ & [65] & 84.51 & 58.18 & 10.92 & 18.39 & NA \\
\hline$\frac{a}{a}$ & Bagging & [60] & $\underline{88.3}$ & 86.8 & $\underline{88.3}$ & 86.4 & $\underline{\mathrm{NA}}$ \\
\hline & AdaBoost & & $\underline{86.8}$ & $\underline{84.6}$ & $\underline{86.8}$ & 84.8 & NA \\
\hline & Long Short Term Memory (LSTM) & & $\underline{85.1}$ & 77.5 & 76.5 & 76.9 & $\underline{\mathrm{NA}}$ \\
\hline & Gated Recurrent Unit (GRU) & & $\underline{88.6}$ & $\underline{83.9}$ & 81.1 & 82.4 & NA \\
\hline 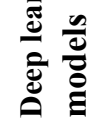 & $\frac{\text { Convolutional Neural Network }}{\underline{(\mathrm{CNN})}}$ & & 87.9 & 85.6 & 82.7 & 84.12 & NA \\
\hline & K-med+GBT+DT+DL+Voting & & 92.40 & 93.23 & 51.34 & 66.22 & 0.14 \\
\hline$\frac{2}{0}$ & K-med+GBT+DT+DL+Bagging & & 92.41 & 93.25 & 51.55 & 66.4 & 0.12 \\
\hline $\bar{\Xi}$ & K-med+GBT+DT+DL+Stacking & & 92.40 & 78.04 & 66.25 & 71.66 & 0.15 \\
\hline : & K-med+GBT+DT+DL+Adaboost & & 92.43 & 78.10 & 66.45 & 71.81 & 0.10 \\
\hline
\end{tabular}

4

5

7

8 


\section{Table $\mathbf{1 0}$ (on next page)}

Comparison with State of the Art Techniques for GitHub Dataset 
1 2

\begin{tabular}{|c|c|c|c|c|c|c|c|}
\hline & Techniques & Reference & Accuracy & Precision & Recall & F-Measure & Standard Dev \\
\hline & FLIC/FDT & [66] & 81.5 & -- & -- & -- & NA \\
\hline$\stackrel{\mathscr{m}}{=}$ & K-Means+DT & [18] & 84.26 & 95.68 & --- & 90.00 & NA \\
\hline 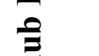 & Bagging + DL & {$[35]$} & 50 & 25 & 50 & 33.33 & NA \\
\hline تَ & AdaBoost+ MLP & & 66.3 & 66.64 & 66.28 & 66.46 & NA \\
\hline 3 & Majority Voting DL+NN+ML & & 66.69 & 67.52 & 66.69 & 67.1 & NA \\
\hline $\bar{g}$ & Bagging + MLP & & 67.57 & 71.54 & 67.57 & 69.5 & NA \\
\hline 河 & Bagging & [58] & 80.8 & 81.88 & 75.28 & 78.44 & NA \\
\hline & AdaBoost & & 73.9 & $\underline{70.46}$ & $\underline{73.74}$ & 72.06 & $\underline{\mathrm{NA}}$ \\
\hline & $\frac{\text { Long Short Term Memory }}{\text { (LSTM) }}$ & & 90.04 & $\underline{84.8}$ & 79.7 & $\underline{82.1}$ & $\underline{\mathrm{NA}}$ \\
\hline & Gated Recurrent Unit (GRU) & & 90.1 & $\underline{85.9}$ & $\underline{83.4}$ & $\underline{84.6}$ & $\underline{\mathrm{NA}}$ \\
\hline 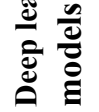 & $\frac{\text { Convolutional Neural Network }}{(\mathrm{CNN})}$ & & 89.8 & 83.1 & $\underline{82.2}$ & $\underline{82.6}$ & $\underline{\mathrm{NA}}$ \\
\hline & K-med+GBT+DT+DL+Voting & & 94.06 & 94.56 & 61.52 & 74.55 & 0.14 \\
\hline 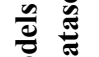 & K-med+GBT+DT+DL+Bagging & & 94.12 & 95.78 & 61.10 & 74.61 & 0.13 \\
\hline 主 & K-med+GBT+DT+DL+Stacking & & 94.65 & 87.33 & 73.12 & 79.59 & 0.11 \\
\hline U. & K-med+GBT+DT+DL+Adaboost & & 94.7 & 87.04 & 75.10 & 80.63 & 0.12 \\
\hline
\end{tabular}

Table 10: Comparison with State of the Art techniques for GitHub Dataset

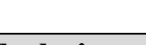

